\title{
Borderline-High Mean Corpuscular Volume Levels Are Associated with Arterial Stiffness among the Apparently Healthy Korean Individuals
}

\author{
Haneul Kwon, Byoungjin Park* \\ Department of Family Medicine, Yonsei University College of Medicine, Seoul, Korea
}

\begin{abstract}
Background: High mean corpuscular volume (MCV) has been implicated in various health problems, such as anemia, liver disease, and thyroid disease. However, the clinical significance of borderline-high MCV is poorly understood in the primary care setting. This study aimed to investigate whether borderline-high MCV was related to arterial stiffness in Korean adults as measured by brachial-ankle pulse wave velocity (baPWV).

Methods: This cross-sectional study comprised 582 participants aged $>30$ years who underwent routine health examinations. Borderline-high MCV was defined as over $95.0 \mathrm{fl}$ ( $>90$ th percentile) after excluding participants with $\mathrm{MCV}$ of $\geq 100 \mathrm{fl}$ or $\leq 80 \mathrm{fl}$, and high baPWV was defined as $>1,600 \mathrm{~cm} / \mathrm{s}$ ( $>90$ th percentile). The odds ratios (ORs) and 95\% confidence intervals (CIs) for high baPWV according to borderline-high MCV were calculated using multiple logistic regression analyses after adjusting for the confounding variables.

Results: The mean age of the study population was $47.8 \pm 11.7$ years, and $56.9 \%$ of the participants were male. Compared to that in the control group, the OR $(95 \% \mathrm{CI})$ of high baPWV in those with a borderline-high MCV was 3.68 (1.39-9.74) after adjusting for age, sex, body mass index, alcohol intake, smoking status, regular exercise, mean blood pressure, fasting plasma glucose, triglyceride, high-density lipoprotein cholesterol, C-reactive protein, $\gamma$-glutamyltransferase, uric acid, hypertension medication, and diabetes medication.

Conclusion: Borderline-high MCV was independently associated with arterial stiffness among apparently healthy Korean individuals.
\end{abstract}

Keywords: Erythrocyte Indices; Pulse Wave Analysis; Vascular Stiffness

Received: January 26, 2019, Revised: July 31, 2019, Accepted: August 4, 2019

*Corresponding Author: Byoungjin Park https://orcid.org/0000-0003-1733-5301

Tel: +82-31-331-8710, Fax: +82-31-331-5551, E-mail: bjpark96@yuhs.ac 


\section{INTRODUCTION}

High mean corpuscular volume (MCV), also known as macrocytosis, is associated with vitamin B12 and folic acid deficiency, liver disease, hyperglycemia, alcoholism, smoking habits, and other pathologic conditions. ${ }^{1,2)}$ Moreover, a high MCV is associated with increased all-cause mortality and cancer mortality in non-anemic cancer-free individuals. ${ }^{3)}$

Pulse wave velocity (PWV) is one of various markers of early arterial wall changes such as arterial wall thickness and stiffness. ${ }^{4)}$ It is defined as the velocity of blood streaming from the heart to peripheral arteries through the aorta and is known to increase with blood vessel stiffness. ${ }^{5,6)}$ Brachial-ankle pulse wave velocity (baPWV) is also strongly correlated with aortic PWV and can be used as a simple, noninvasive, and automatic method to measure arterial stiffness in the primary care setting. ${ }^{\text {.) }}$ Previous studies have shown that increased PWV is independently associated with cardiovascular disease (CVD) incidence and mortality. Moreover, high baPWV was a strong predictor of early carotid atherosclerosis. ${ }^{8)} \mathrm{A}$ baPWV of $>1,400 \mathrm{~cm} / \mathrm{s}$ is an independent risk factor for moderate risk ( $\geq 10 \%$ ) stratification according to the Framingham risk scoring system, and a baPWV of $>1,600 \mathrm{~cm} / \mathrm{s}$ is an independent factor for high risk stratification according to the European systematic coronary risk evaluation (SCORE) system. ${ }^{9}$

Although high MCV has been implicated in various health problems, the clinical significance of borderline-high MCV is poorly understood among apparently healthy Korean individuals in the primary care setting. Therefore, this study aimed to examine whether borderline-high MCV is related to arterial stiffness in Korean adults as measured using baPWV.

\section{METHODS}

\section{Study Population}

Medical records of 641 participants who voluntarily underwent a medical examination including baPWV measurement at the Health Promotion Center of Gangnam Severance Hospital in Seoul, Korea, between October 2007 and July 2010 were reviewed. Participants meeting any of the following criteria were excluded ( $\mathrm{n}=59)$ : $\mathrm{MCV}, \geq 100$ $\mathrm{fl}$ or $\leq 80 \mathrm{fl}$; white blood cell (WBC) count, $\geq 10.0\left(\times 10^{3}\right.$ cells $\left./ \mu \mathrm{L}\right)$; and high-sensitivity C-reactive protein (hsCRP), $\geq 3.0 \mathrm{mg} / \mathrm{L}$. After excluding non-eligible participants, 582 participants were finally enrolled into the final analysis. This study was conducted in accordance with the ethical principles of the Declaration of Helsinki and was approved by the Institutional Review Board of Yonsei University College of Medicine, Seoul, Korea (IRB approval no., 3-2019-0098). Informed consent was obtained from all individual participants included in the study.

\section{Data Collection}

Participants were asked about their lifestyle, including smoking habits, alcohol consumption, and physical activity (more or less than 2 times per week), as well as their medical conditions with the date of diagno- sis and current medications. Trained staff reviewed the completed questionnaires and recorded the responses into a database. Participants were classified as non-smokers, ex-smokers, or current smokers. Alcohol drinking was defined as consumption of more than 2 times per week. Regular exercise was defined as exercise of more than 2 times per week. Body mass index (BMI) was calculated as weight divided by height squared $\left(\mathrm{kg} / \mathrm{m}^{2}\right)$. Blood samples were collected after a 12-hour overnight fast. WBC counts and red blood cell indices were quantified by an automated blood cell counter (ADVIA 120; Bayer Corp., Tarrytown, NY, USA). Borderline-high MCV was defined as $\geq 95.0 \mathrm{fl}$ and $<100 \mathrm{fl}$ based on previous epidemiological studies ( $>90 \mathrm{th}$ percentile). ${ }^{1,3)}$ Fasting plasma glucose, total cholesterol, triglycerides, high-density lipoprotein (HDL) cholesterol, hsCRP, $\gamma$-glutamyltransferase, and uric acid were measured using a Hitachi 7600-110 Chemistry Autoanalyzer (Hitachi, Tokyo, Japan). The mean blood pressure was calculated using the following equation: (systolic blood pressure+ diastolic blood pressure $\times 2) / 3$.

\section{Brachial-Ankle Pulse Wave Velocity Measurement}

As previously reported, ${ }^{5,8,10,11)}$ baPWV was measured and calculated using an automatic waveform analyzer (model BP-203RPE; Colin Co., Komaki, Japan). This instrument simultaneously recorded venous blood pressure, phonocardiogram, electrocardiogram, and arterial blood pressure in both upper arms and ankles. Participants were examined in supine position after at least 10 minutes of rest. Pneumatic cuffs were connected to sensors and wrapped around both upper arms and ankles. Waveforms were obtained from brachial and tibial arteries. For each measurement, 10-second sample times with automatic gain analysis and quality adjustment were obtained. The time interval $(\Delta \mathrm{T})$ between the arm and ankle distance was defined as the pulse transit time between the brachial and tibial arterial pressure waveforms. $L$ was defined as the difference between the distance from the heart to the upper arm and the distance from the heart to the ankle and was automatically estimated based on participants' heights. BaPWV was calculated using the following equation: baPWV=L/ $\Delta \mathrm{T}$. BaP$\mathrm{WV}$ of $>1,400 \mathrm{~cm} / \mathrm{s}$ and $>1,600 \mathrm{~cm} / \mathrm{s}$ are known independent variables for risk stratification according to the Framingham risk and SCORE risk scores, respectively. ${ }^{7,9)}$ In this regard, high baPWV, a surrogate indicator for arterial stiffness, was defined as $>1,600 \mathrm{~cm} / \mathrm{s}$ in this study, which corresponded to the 90th percentile.

\section{Statistical Analysis}

The Student t-test and chi-square test were used to compare demographic and biochemical characteristics of the study population according to borderline-high MCV for continuous and categorical variables, respectively. Univariate analysis was performed to evaluate the relationship between arterial stiffness and several cardiovascular risk factors. Analysis of covariance was also used to verify differences in mean baPWV according to the borderline-high MCV after adjusting for age, sex, BMI, hsCRP, hypertension medication, and diabetes medication. 
The odds ratios (ORs) for high baPWV were calculated using multivariate logistic regression analysis after adjusting for confounding variables according to borderline-high MCV. All analyses were conducted using SAS statistical software ver. 9.4 (SAS Institute Inc., Cary, NC, USA). All statistical tests were two-sided, and statistical significance was determined at a P-value of $<0.05$.

\section{RESULTS}

Table 1 shows the clinical characteristics of the study population (330 men and 252 women). The mean age and BMI were $47.8 \pm 11.7$ years and $23.8 \pm 2.9 \mathrm{~kg} / \mathrm{cm}^{2}$, respectively. The mean systolic and diastolic blood pressure were 119.5 $\pm 15.2 \mathrm{~mm} \mathrm{Hg}$ and $73.1 \pm 11.9 \mathrm{~mm} \mathrm{Hg}$, respec- tively. The proportion of participants with borderline-high MCV was $10.5 \%$. Compared with controls, age, male sex, and $\gamma$-glutamyltransferase were higher in the borderline-high MCV group.

Table 2 shows the univariate analysis between high baPWV and some indicators for cardiovascular risk. BaPWV was found to be significantly associated with age (OR, 1.13; $\mathrm{P}<0.001)$, BMI (OR, 1.17; $\mathrm{P}<0.001$ ), systolic blood pressure ( $\mathrm{OR}, 1.08 ; \mathrm{P}<0.001)$, diastolic blood pressure (OR, 1.11; $\mathrm{P}<0.001)$, fasting plasma glucose (OR, 1.02; $\mathrm{P}<0.001)$, hsCRP (OR, 1.43; $\mathrm{P}<0.001)$, and borderline-high $\mathrm{MCV}(\mathrm{OR}$, 3.05; $\mathrm{P}<0.001)$.

Figure 1 shows the means and standard errors of baPWV $(\mathrm{cm} / \mathrm{s})$ according to borderline-high MCV after adjusting for age, sex, BMI, hsCRP, hypertension medication, and diabetes medication. The mean

Table 1. Characteristics of the study population

\begin{tabular}{|c|c|c|c|c|}
\hline Characteristic & Total $(n=582)$ & Control $(n=532)$ & $\begin{array}{l}\text { Borderline-high mean } \\
\text { corpuscular volume }(n=50)\end{array}$ & P-value \\
\hline Age (y) & $47.8 \pm 11.7$ & $47.3 \pm 11.7$ & $52.5 \pm 10.5$ & 0.002 \\
\hline Body mass index $\left(\mathrm{kg} / \mathrm{m}^{2}\right)$ & $23.8 \pm 2.9$ & $23.8 \pm 2.9$ & $23.5 \pm 2.7$ & 0.586 \\
\hline Male gender & $331(56.9)$ & $295(55.5)$ & $36(72.0)$ & 0.023 \\
\hline Current smoker & $132(22.7)$ & $120(22.6)$ & $12(24.0)$ & 0.815 \\
\hline Alcohol consumption & $420(71.2)$ & $379(71.2)$ & $41(82.0)$ & 0.104 \\
\hline Regular exercise & $344(59.1)$ & $311(58.5)$ & $33(66.0)$ & 0.299 \\
\hline Systolic blood pressure (mm Hg) & $119.5 \pm 15.2$ & $119.4 \pm 15.3$ & $120.8 \pm 14.4$ & 0.527 \\
\hline Diastolic blood pressure (mm Hg) & $73.1 \pm 11.9$ & $72.9 \pm 12.0$ & $75.5 \pm 10.9$ & 0.145 \\
\hline Fasting plasma glucose (mg/dL) & $96.2 \pm 18.5$ & $96.1 \pm 18.9$ & $96.7 \pm 14.3$ & 0.784 \\
\hline Triglyceride (mg/dL) & $113.7 \pm 94.7$ & $112.5 \pm 90.2$ & $127.1 \pm 134.0$ & 0.453 \\
\hline High-density lipoprotein cholesterol (mg/dL) & $48.1 \pm 12.6$ & $47.9 \pm 12.5$ & $50.1 \pm 13.9$ & 0.226 \\
\hline White blood cells $\left(\times 10^{3}\right.$ cell $\left./ \mu \mathrm{L}\right)$ & $5.5 \pm 1.4$ & $5.5 \pm 1.3$ & $5.5 \pm 1.6$ & 0.903 \\
\hline C-reactive protein (mg/L) & $0.6 \pm 0.6$ & $0.6 \pm 0.6$ & $0.6 \pm 0.7$ & 0.694 \\
\hline$\gamma$-glutamyltransferase (U/L) & $24.4 \pm 16.0$ & $23.7 \pm 15.3$ & $32.3 \pm 20.6$ & 0.005 \\
\hline Uric acid (mg/dL) & $5.2 \pm 1.4$ & $5.2 \pm 1.4$ & $5.4 \pm 1.3$ & 0.198 \\
\hline Hemoglobin (d/dL) & $14.6 \pm 1.4$ & $14.6 \pm 1.4$ & $14.9 \pm 1.2$ & 0.197 \\
\hline Hypertension medication & $73(12.5)$ & $70(13.2)$ & $3(6.0)$ & 0.144 \\
\hline Diabetes medication & $24(4.1)$ & $23(4.3)$ & $1(2.0)$ & 0.429 \\
\hline
\end{tabular}

Values are presented as mean \pm standard deviation or number (\%), unless otherwise stated.

Table 2. Univariate analysis of factors associated with high brachial-ankle pulse wave velocity

\begin{tabular}{lcc}
\hline \multirow{2}{*}{ Variable } & \multicolumn{2}{c}{ High brachial-ankle pulse wave velocity } \\
\cline { 2 - 3 } & \multicolumn{1}{c}{ Odds ratio } & P-value \\
\hline Age $(\mathrm{y})$ & $1.13(1.09-1.16)$ & $<0.001$ \\
Body mass index $\left(\mathrm{kg} / \mathrm{m}^{2}\right)$ & $1.17(1.07-1.28)$ & $<0.001$ \\
Systolic blood pressure $(\mathrm{mm} \mathrm{Hg})$ & $1.08(1.06-1.10)$ & $<0.001$ \\
Diastolic blood pressure $(\mathrm{mm} \mathrm{Hg})$ & $1.11(1.08-1.14)$ & $<0.001$ \\
Fasting plasma glucose $(\mathrm{mg} / \mathrm{dL})$ & $1.02(1.00-1.03)$ & 0.007 \\
Triglyceride $(\mathrm{mg} / \mathrm{dL})$ & $1.00(1.00-1.01)$ & 0.016 \\
High-density lipoprotein cholesterol & $0.98(0.96-1.00)$ & 0.082 \\
$\quad$ & & \\
C-reactive protein (mg/L) & $1.43(1.11-1.84)$ & 0.005 \\
$\gamma$-glutamyltransferase (U/L) & $1.01(0.99-1.02)$ & 0.262 \\
Uric acid (mg/dL) & $1.01(0.84-1.22)$ & 0.885 \\
Borderline-high mean corpuscular & $3.05(1.53-6.08)$ & 0.001 \\
$\quad$ volume (yes or no) & & \\
\hline
\end{tabular}

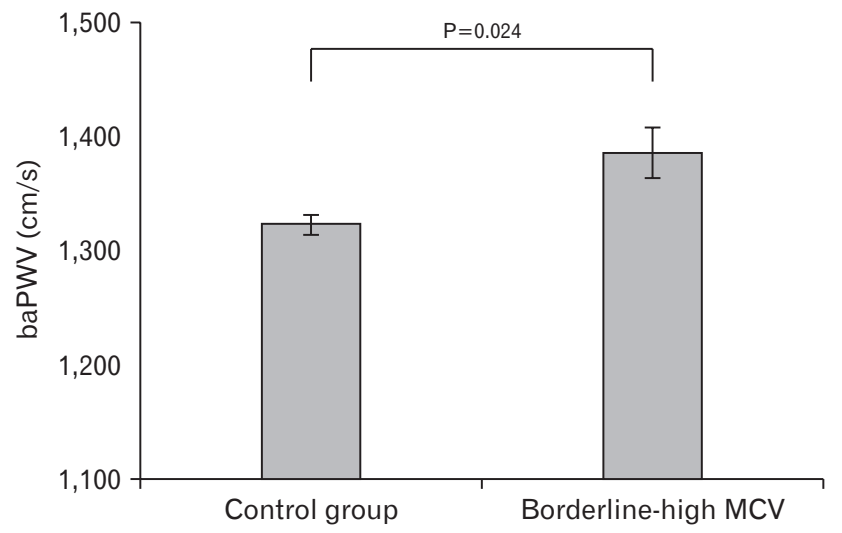

Figure 1. Mean baPWV according to the presence of borderline-high MCV as determined by analysis of covariance analysis adjusted for age, sex, body mass index, high-sensitivity C-reactive protein, hypertension medication, and diabetes medication. BaPWV, brachial-ankle pulse wave velocity; MCV, mean corpuscular volume. 
Table 3. Multiple logistic regression analysis showing the independent contribution of borderline-high MCV to high brachial-ankle pulse wave velocity

\begin{tabular}{|c|c|c|c|c|c|c|}
\hline & \multicolumn{2}{|c|}{ Model 1} & \multicolumn{2}{|c|}{ Model 2} & \multicolumn{2}{|c|}{ Model 3} \\
\hline & OR (95\% Cl) & P-value & OR (95\% Cl) & P-value & $\mathrm{OR}(95 \% \mathrm{Cl})$ & P-value \\
\hline Borderline-high MCV & $3.05(1.53-6.08)$ & 0.001 & $3.08(1.19-7.98)$ & 0.020 & $3.21(1.22-8.48)$ & 0.018 \\
\hline
\end{tabular}

Model 1: unadjusted; model 2: adjusted for age, sex, BMl, alcohol intake, smoking status, regular exercise, SBP, DBP, FPG, triglyceride, HDL-cholesterol, WBC count, CRP, $\gamma$-glutamyltransferase, and uric acid; model 3: adjusted for age, sex, BMl, alcohol intake, smoking status, regular exercise, SBP, DBP, FPG, triglyceride, HDL-cholesterol, WBC count, CRP, $\gamma$-glutamyltransferase, uric acid, hypertension medication, and diabetes medication.

MCV, mean corpuscular volume; OR, odds ratio; Cl, confidence interval; BMI, body mass index; SBP, systolic blood pressure; DBP, diastolic blood pressure; FPG, fasting plasma glucose; HDL, high-density lipoprotein; WBC, white blood cell; CRP, C-reactive protein.

baPWV in the borderline-high MCV group was significantly higher than that in the control group $(1,386.2 \pm 22.7 \mathrm{~mm} \mathrm{Hg}$ versus $1,322.7 \pm 8.7$ $\mathrm{mm} \mathrm{Hg}, \mathrm{P}=0.024$ ).

Table 3 shows the ORs for high PWV according to the borderlinehigh MCV. The OR (95\% CI) of the borderline-high MCV group was 3.21 (1.22-8.48) after adjusting for age, sex, BMI, alcohol intake, smoking status, regular exercise, systolic blood pressure, diastolic blood pressure, fasting plasma glucose, triglyceride, HDL-cholesterol, WBC count, C-reactive protein, $\gamma$-glutamyltransferase, and uric acid, hypertension medication, and diabetes medication.

\section{DISCUSSION}

In this cross-sectional study, borderline-high MCV was independently associated with high baPWV. This effect remained even after adjusting for potential confounding factors, including the presence of drugs that could influence the vascular function. Although the reference value for baPWV is different according to age and blood pressure categories, ${ }^{12,13)}$ a baPWV of $>1,400 \mathrm{~cm} / \mathrm{s}$ has been shown as a useful predictor of CVD, ${ }^{7)}$ and $>1,800 \mathrm{~cm} / \mathrm{s}$ may be a marker of target organ damage. ${ }^{14)}$ Previous Korean studies showed that a baPWV of $>1,600 \mathrm{~cm} / \mathrm{s}$ was an independent risk factor for greater atherosclerotic disease complexity based on the virtual histology intravascular ultrasound analysis and high risk stratification based on the SCORE model. ${ }^{9,15)}$

Although underlying mechanisms of increased baPWV were not investigated, some biological mechanisms may help explain the association between borderline-high MCV and arterial stiffness. First, increased MCV reflects folate and vitamin B12 deficiencies, which can lead to high homocysteine level, a cardiovascular risk factor. Folate and vitamin B12 play an important role in regulating the conversion of homocysteine. Homocysteine concentrations are strongly negatively correlated with folate and vitamin B12 levels. ${ }^{16,17)}$ Homocysteine induces proliferation of smooth muscle cells in the artery, resulting in impaired vascular endothelial function and blood coagulability. High homocysteine level is thought to contribute to atherosclerosis and increased thickness and blood vessel stiffness. ${ }^{10)}$ Accordingly, lack of folate and vitamin B12 induces elevated MCV and homocysteine levels, resulting in elevated arterial stiffness. Second, elevated MCV is an indicator of liver function deterioration, which might be closely related to CVD development. Macrocytosis is commonly found in various liver diseases. In individuals with liver disease, the membrane lipid com- position changes, affecting the membrane surface area, leading to increased MCV. This mechanism depends on the pathogenesis and severity of liver disease. ${ }^{2)}$ Moreover, elevated serum $\gamma$-glutamyltransferase level is strongly associated with increased oxidative stress. ${ }^{18,19)}$ Increased oxidative stress induced by decreased liver function is positively correlated with CVD development. ${ }^{20)}$ Thus, decreased liver function seems to manifest in elevated MCV as well as arterial stiffness. Finally, elevated MCV implies thyroid dysfunction, which could also be associated with arterial stiffness. Thyroid hormone is involved in the regulation of hemoglobin synthesis. MCV values were significantly increased in both overt and subclinical hypothyroidism. ${ }^{21)}$ Moreover, hypothyroidism led to increased vascular resistance and arterial wall thickness, which could be attributed to lipid abnormalities, homocysteine levels, and abnormal coagulability. ${ }^{22)}$ Therefore, elevated MCV and arterial stiffness might be caused by thyroid dysfunction. Therefore, our findings indicate borderline-high MCV may be accompanied by several unfavorable metabolic alterations, which can be implicated in increased arterial stiffness.

Several limitations should be acknowledged in this study. First, the causality cannot be concluded due to the cross-sectional design of the study. Moreover, other confounding factors affecting baPWV, such as thyroid disease, homocysteine levels, and dyslipidemia medication, were not considered because these indicators were not fully measured at the beginning of this study. Study participants were volunteers visiting for a health promotion screening at a single hospital. Thus, the study population appeared to be slightly healthier than communitybased cohorts and may not be representative of the general population in Korea, and selection bias may have affected the results.

In conclusion, we found that borderline-high MCV was independently associated with arterial stiffness among apparently healthy Korean individuals. Further larger-scale prospective cohort studies are warranted to validate these associations in the future.

\section{CONFLICT OF INTEREST}

No potential conflict of interest relevant to this article was reported.

\section{ORCID}

Haneul Kwon: https://orcid.org/0000-0002-7072-0711

Byoungjin Park: https://orcid.org/0000-0003-1733-5301 


\section{REFERENCES}

1. Yang SH. Relationship between mean corpuscular volume and liver function test. Korean J Clin Lab Sci 1996;28:134-9.

2. Maruyama S, Hirayama C, Yamamoto S, Koda M, Udagawa A, Kadowaki Y, et al. Red blood cell status in alcoholic and non-alcoholic liver disease. J Lab Clin Med 2001;138:332-7.

3. Yoon HJ, Kim K, Nam YS, Yun JM, Park M. Mean corpuscular volume levels and all-cause and liver cancer mortality. Clin Chem Lab Med 2016;54:1247-57.

4. Park BJ, Shim JY, Lee HR, Lee JH, Jung DH, Kim HB, et al. Relationship of neutrophil-lymphocyte ratio with arterial stiffness and coronary calcium score. Clin Chim Acta 2011;412:925-9.

5. Park BJ, Chun HY, Han AR, Lim J, Lee DC, Shim JY, et al. Relationship of serum homocysteine with brachial-ankle pulse wave velocity in middle aged women. Korean J Fam Med 2009;30:457-63.

6. Kim GL, Hwang HR, Kim YJ, Lee SY, Lee JG, Jeong DW, et al. The association of body fat and arterial stiffness using the brachial-ankle pulse wave velocity. Korean J Fam Med 2018;39:347-54.

7. Yamashina A, Tomiyama H, Arai T, Hirose K, Koji Y, Hirayama Y, et al. Brachial-ankle pulse wave velocity as a marker of atherosclerotic vascular damage and cardiovascular risk. Hypertens Res 2003;26:615-22.

8. Kubozono T, Miyata M, Kawasoe S, Ojima S, Yoshifuku S, Miyahara H, et al. High pulse wave velocity has a strong impact on early carotid atherosclerosis in a Japanese general male population. Circ J 2017;81: 310-5.

9. Kim YK, Kim D. The relation of pulse wave velocity with Framingham risk score and SCORE risk score. Korean Circ J 2005;35:22-9.

10. Yoon KS, Kim SW, Choi HJ. Association between plasma homocysteine level and brachial-ankle pulse wave velocity in Korean adults. Korean J Fam Med 2009;30:46-54.

11. Park BJ, Lee HR, Shim JY, Lee JH, Jung DH, Lee YJ. Association between resting heart rate and arterial stiffness in Korean adults. Arch Cardiovasc Dis 2010;103:246-52.

12. Yiming G, Zhou X, Lv W, Peng Y, Zhang W, Cheng X, et al. Reference values of brachial-ankle pulse wave velocity according to age and blood pressure in a central Asia population. PLoS One 2017;12: e0171737.

13. Reference Values for Arterial Stiffness' Collaboration. Determinants of pulse wave velocity in healthy people and in the presence of cardiovascular risk factors: 'establishing normal and reference values.' Eur Heart J 2010;31:2338-50.

14. Matsumoto C, Tomiyama H, Yamada J, Yoshida M, Shiina K, Yamashina A. Brachial-ankle pulse wave velocity as a marker of subclinical organ damage in middle-aged patients with hypertension. J Cardiol 2008;51:163-70.

15. Kwon JE, Mintz GS, Kim SW, Oh MS, Min YJ, Kim HK, et al. Relationship between coronary artery plaque composition by virtual histology intravascular ultrasound analysis and brachial-ankle pulse wave velocity in patients with coronary artery disease. Coron Artery Dis 2011; 22:565-9.

16. Aslinia F, Mazza JJ, Yale SH. Megaloblastic anemia and other causes of macrocytosis. Clin Med Res 2006;4:236-41.

17. Ma Y, Peng D, Liu C, Huang C, Luo J. Serum high concentrations of homocysteine and low levels of folic acid and vitamin $\mathrm{B}(12)$ are significantly correlated with the categories of coronary artery diseases. BMC Cardiovasc Disord 2017;17:37.

18. Laurent S, Boutouyrie P, Asmar R, Gautier I, Laloux B, Guize L, et al. Aortic stiffness is an independent predictor of all-cause and cardiovascular mortality in hypertensive patients. Hypertension 2001;37:123641.

19. Targher G, Byrne CD. Circulating markers of liver function and cardiovascular disease risk. Arterioscler Thromb Vasc Biol 2015;35:2290-6.

20. Park BJ, Lee YJ, Lee HR. Chronic liver inflammation: clinical implications beyond alcoholic liver disease. World J Gastroenterol 2014;20: 2168-75.

21. Bashir H, Bhat MH, Farooq R, Majid S, Shoib S, Hamid R, et al. Comparison of hematological parameters in untreated and treated subclinical hypothyroidism and primary hypothyroidism patients. Med J Islam Repub Iran 2012;26:172-8.

22. Dagre AG, Lekakis JP, Papaioannou TG, Papamichael CM, Koutras DA, Stamatelopoulos SF, et al. Arterial stiffness is increased in subjects with hypothyroidism. Int J Cardiol 2005;103:1-6. 